\title{
EDITORIAL
}

\section{Abdominal Hydatidosis: Surgical Perspectives}

Hydatid disease or Echinococcosis is a zoonotic disease (Krabbe and Finsen 1862) caused by the larva of Echinococcus species and is one of the oldest known parasitic diseases to man. The disease has a worldwide distribution and is also well recognised and documented in the Indian subcontinent. The disease is caused by the larval stage of Echinococcosis. Humans are accidental intermediate/dead-end hosts whereas animals can be both intermediate and definitive hosts. The two main types of hydatid diseases are caused by E.granulosus and E.multilocularis, former being the most common type in humans and liver is the most commonly affected organ ${ }^{[1]}$. The other types include E.vogeli, E.oligarthus, E.shiquicus, E.ortleppi and E.equinus. In humans, 50-75\% of the cysts occur in liver, $25 \%$ in lungs and $5-10 \%$ distribute along the arterial system ${ }^{[2]}$

Diagnosis of human hydatidosis used to be a challenge in the remote past due to lack of specific investigations and hence more of such patients would present in the emergency department with one or the other complications of abdominal hydatid (such as cyst rupture, abscess, peritonitis etc.), be it in liver, spleen, peritoneal cavity or elsewhere and hence, were subjected to emergency surgeries. However, in recent years, the diagnosis of this disease has been quite easy and even early due to availability of various radiological investigations such as ultrasonography, computed tomography scan and MRI, the latter being of more value in cardiac and disseminated disease and MRI is preferred over $\mathrm{CT}$ whenever possible because of its higher sensitivity for the characterization of the lesion matrix ${ }^{[3]}$. Also, MRI is more specific than $\mathrm{CT}$ in diagnosing cystobiliary communications, where intracystic fat density is present $t^{[4]}$. Recent immunological assays have been very useful in diagnosing the seropositive cases well in time and their titers guide us in monitoring the response to treatment be it drugs or surgery or both whatever is offered. However, post surgery' even with complete removal of the disease, blood titers may decrease very slowly over months to years, and, therefore, it is the progressive rising titers and not the positive serological tests which are indicative of any recurrence of the disease.
The availability of various drugs (benzimidazoles, praziquantel) have proven to be effective in sterilizing the cysts in most cases if given preoperatively and to reduce the recurrence rates if continued postoperatively, keeping a close watch on the liver functions as most of these antihelminthics are hepatotoxic agents. Such adjuvant therapy also helps in reducing the complications of hydatid cysts already mentioned. Initially, benzimidazoles were given in cycles with an interruption in between. However, currently, treatment interruption is not recommended ${ }^{[5]}$. Also, there is no clear-cut consensus on the duration of preoperative albendazole therapy. There is literature suggesting treatment benefit if the drug is started anywhere between 1 month and 4 days before surgery. In a study which was conducted at SKIMS Srinagar, 12 weeks preoperative and 12 weeks postoperative albendazole therapy was found to be quite effective ${ }^{[6]}$. According to a review given in journal "Infectious disorders-Drug targets" by Dehkordi AB et al, it could be concluded that treatment with albendazole may be associated with prevention from recurrence and reduction of the size and death of the cysts.

Management options for liver hydatid cysts include operative methods which include conservative (such as capsulorrhaphy, captonage, introflexion, open endocystectomy with or without omentoplasty, or external tube drainage of infected or communicating cysts) and radical procedures (total pericystectomy /capsulectomy / cystopericystectomy or liver resection), and non-operative methods which include chemotherapy and percutaneous treatment (PAIR and PEVAC) for uncomplicated cysts. Whatever the management protocol adopted, the objectives of treatment are getting rid of the entire parasitic load, closure/removal of the residual cavity and management of any fistulae, if present. The use of alcohol as a scolicidal and sclerosing agent during percutaneous treatment (PAIR procedure) of liver hydatid cysts has been observed to achieve high success rates and low rates of recurrence and complications and can be considered alternative to surgical procedures. ${ }^{[7]}$. Laparoscopy, introduced in the early 90 's as a modality for surgical interventions has grown a lot since then. Most of the uncomplicated liver hydatids are managed 
well by laparoscopy by experienced surgeons which has changed the surgical management particularly for simple cysts as well as some cases of complicated cysts. Minimal invasive management using aspiration and laparoscopic intervention is an alternative to open surgery because of its ability to prevent spillage and thus minimise recurrences and is associated with less morbidity, mortality and recurrence rate in comparison to open technique. ${ }^{[8]}$. In a separate study, it was confirmed that laparoscopy in hepatic hydatidosis is safe and effective and has advantages in terms of lesser complications and hospital stay. Modifications in surgeries have given surgeons an edge in the overall management of abdominal hydatidosis but some postoperative complications of cyst management such as prolonged and persistent postoperative cyst cavity drainage is managed successfully by endoscopic methods including papillotomy or rarely by biliary stenting. Omentoplasty is one of the safest methods to manage the cyst cavity where omentum is mobilized from the transeverse colon to obliterate and pack the cavity. In case the cavity is too large to be managed by omentum, a tube drain is placed into the cavity alongside the mobilized omentum. Other techniques such as capitonage and capsulorrhaphy are used somewhat rarely. Complicated hydatid liver cysts can be successfully managed surgically (omentoplasty with or without internal/external drainage) with good long term results ${ }^{[9]}$. Complications of hydatid cysts such as rupture, anaphylaxis, abscess, fistulas, internal rupture into biliary tree, stomach, peritoneal cavity can give rise to disseminated abdominal hydatids which is very difficult to manage and can have a very high morbidity and mortality. Cyst rupture into thoracic cavity is usually managed by treatment of cyst with hepatodiaphragmatic disconnection and eventually a restoration of the diaphragm after treating thoracic disease and securing diaphragm and biliary tree. Similarly, in pericardial rupture cystopericystectomy is the gold standard procedure. Involvement of peritoneal cavity mandates peritoneal washing with irrigation of peritoneal cavity with sufficient amounts of scolicidal agents. ${ }^{[10]}$ Successful early diagnosis and management can avoid all these complications.

All patients of abdominal hydatidosis have to be on regular follow up with a close watch on antibody titers, liver function tests, complete blood counts and abdominal ultrasonography when needed as these patients are put on benzimidazoles and praziquantel to prevent recurrence of the disease.
Ajaz Ahmad Malik,
M.S, FAIS, FICS, FMAS, MAMS, FIAGES, FACS
Professor \& Division Head
Deptt. Of General and Minimal Invasive Surgery
SKIMS, Srinagar

\section{REFERENCES}

1. Shaw JM, Bornman PC, Krige JEJ. "Hydatid disease of the liver." Soyht Afr J Surg SUID-Afr Tydskr Vir Chir 44, 2006: 70-72, 74-77,.

2. Gunay K, Korhan MD, Taviloglu, et al. "Traumatic rupture of hydatid cysts; a 12 year experience from endemic region." J Trauma, 1999: 46;164-67.

3. Hosch W. "Metabolic viability assessment of cystic echinococcus using high field 1H MRS of cyst contents." NMR Biomed, 2008: 21:734-754.

4. C, Basaran. "Fat containing lesions of the liver: cross sectional imaging findings with emphasis on MRI." AJR Am JRoentgenol, 2005: 184:1103-1110.

5. E, Brunetti. "Expert consensus for the diagnosis and treatment of cystic and alveolar echinococcosis in humans." acta Trop, 2010: 114:1-16.

6. Shams ul Bari, Naqash MM, Malik AA." Outcome of albendazole therapy in management of hepatic hydatid disease." International journal of research in medical sciences, 2016: 3549-3553

7. Bakdik S, Arslan S, Oncu F, et al. "Percutaneous treatment of hepatic cystic echinococcus: success of alcohol as a single endocavitary agent in PAIR, catheterization and modified catheterization techniques." La Radiolgia Medica, 2018: 153-160.

8. Darbari A, Jauhari A, Shirvastava A. "Management of hepatic hydatidosis by open versus laparoscopic surgery." International journal of Research in Medical Sciences, 2014: Vol 2, No 4.

9. Ajaz A Malik, Shams ul Bari, Ruquia Amin, Masooda Jan. "Surgical Management of Complicated Hydatid Cysts of Liver." World Journal of Gastrointestinal Surgery, 2010: 2(3):78-84

10. Yiallourou AI, Nastos C, Theodoraki K, et al. "Surgical Management of Major Complications of Hydatid Cysts of Liver- A review of literature." Annals of clinical cytology and pathology, 2017:3(1): 1049. 